\title{
Modern approaches to noninvasive diagnosis of malignant transformation of endometriosis
}

\author{
HIROSHI KOBAYASHI, YUKI YAMADA, NAOKI KAWAHARA, KENJI OGAWA and CHIHARU YOSHIMOTO \\ Department of Obstetrics and Gynecology, Nara Medical University, Kashihara, Nara 634-8522, Japan
}

Received July 28, 2018; Accepted October 26, 2018

DOI: $10.3892 / \mathrm{ol} .2018 .9721$

\begin{abstract}
Endometriosis-associated ovarian cancer (EAOC) is a rare entity and has highly variable morphological presentations. Mural nodules can be seen in EAOC and benign ovarian endometrioma $(\mathrm{OE})$, which causes a diagnostic dilemma. The differential diagnosis between early-stage EAOC with predominantly cystic appearances and benign OE remains a challenge for physicians. This study will summarize recent knowledge of diagnosis of malignant transformation of endometriosis that have been studied through an innovative approach based on a wide array of novel technologies. Using PubMed database, we focused on the biochemical and technical advancement in the differential diagnosis of benign and malignant endometriosis. Compared with the subjects with benign $\mathrm{OE}$, cyst fluid hemoglobin and iron-related compounds levels were significantly lower in patients with EAOC. This observation opens up the possibility of early diagnosis before morphological variations are captured through ultrasonographic and magnetic resonance (MR) imaging diagnosis. The metallobiology technology offers one solution to this challenge. We discuss the noninvasive diagnosis of EAOC via various imaging methods, including electronic absorption spectroscopy, near infrared approach and MR transverse relaxometry. Special emphasis is given to recent advances in the noninvasive imaging modalities.
\end{abstract}

\section{Introduction}

Ovarian cancer is known to develop in approximately $1 \%$ of women with endometriosis (1). Endometriosis might be related to an increased risk of EAOC, but the underlying mechanism remains unclear (2). Current screening modalities for detecting differences between patients with benign ovarian endometrioma (OE) and EAOC include transvaginal ultrasounds

Correspondence to: Professor Hiroshi Kobayashi, Department of Obstetrics and Gynecology, Nara Medical University, 840 Shijo-cho, Kashihara, Nara 634-8522, Japan

E-mail: yuki0528@naramed-u.ac.jp

Key words: endometriosis, malignant transformation, noninvasive diagnosis, metallobiology and serum CA125 levels. CA125 is the most commonly used serum marker to predict the presence of ovarian cancer, but the specificity of CA125 is low (3). Recent retrospective study demonstrated that the preoperative CA125 value was not useful for detecting patients with malignant transformation of endometriosis (4). So far, no sensitive serum biomarker has been characterized. Furthermore, the important preoperative findings for a diagnosis of malignant change is the presence of endometriotic lesions with worrisome features, including a mural nodule, a solid mass, a thickened wall, and a cyst size of $>7 \sim 8 \mathrm{~cm}$, or rapid cyst growth at ultrasonography (1). If EAOC shows a lack of mural nodules in its early stages, this tumor causes great diagnostic difficulties. At present, early and accurate clinical noninvasive prediction of malignant transformation remains challenging. We will summarize the accumulated current status of knowledge of recent advances in the various imaging modalities for the noninvasive diagnosis of EAOC. Growth in current knowledge of carcinogenesis facilitates strategies for early diagnosis, appropriate management, and disease monitoring.

\section{Materials and methods}

A computerized literature search was conducted to identify relevant studies reported in the English language. We collected a comprehensive literature search from PubMed and Embase database up to April 2018, combining the keywords 'endometriosis', 'endometriosis-associated ovarian cancer', 'endometrioid carcinoma', 'clear cell carcinoma', 'diagnosis', 'discrimination', 'hemoglobin' and 'iron'. A variety of combinations of these terms were used, depending on which database was searched. Furthermore, the references of each article were searched to identify potentially relevant studies. Publications of original studies and review papers were included, while those documenting opinions, points of view or anecdotes were discarded. The flow chart of the literature search is presented in Fig. 1.

Noninvasive techniques and novel modalities for discrimination of EAOC from benign $O E$. The aim of this review was to discuss an effective and noninvasive diagnostic tool for discrimination between EAOC and benign OE. EAOC is thought to arise from endometriosis and should be considered in the differential diagnosis of a pelvic mass (2). Most important is to make the discrimination between malignant and benign 
lesions preoperatively. Ultrasound is used to differentiate solid lesions from simple cysts. Typical ultrasound features of EAOC include well-circumscribed masses with mural nodules resembling ‘xiaolongbao' (Personal communication from Prof. Dr. Mikami Y. Kumamoto University). However, the overlapping appearances of benign and malignant lesions make ultrasound less useful in differentiating malignant lesions, resulting in a large number of surgery for benign tumors (5). Therefore, ultrasonic morphological features are not relevant for the discrimination between EAOC and benign OE with mural nodules. Zhou and Hua (6), recently demonstrated that times of pregnancy $>1.5$, tumor size $>8.3 \mathrm{~cm}$, and the presence of uterine leiomyoma and multiple foci of endometriosis were independent risk factors for EAOC. Tanase et al (5), identified the patient demographics, clinical features and preoperative MR imaging characteristics helpful to the differential diagnosis between EAOC and benign OE with mural nodules. A majority $(\sim 80 \%)$ of the mural nodular lesions of benign $\mathrm{OE}$ showed the pattern described as 'cyst with retracted blood clots'. Malignant transformation typically manifests as cystic lesions with mural nodules, with various (low, intermediate and high) signal intensities on T1-weighted images, high-signal intensity on T2-weighted images, and a lower proportion of shading in women undergoing screening MR imaging. Malignant mural nodules was also found in the anterior location of the cyst (5). When compared to subjects with benign nodules, the patients with malignant mural nodules were older ( $>43$ years), had larger cyst diameters $(>7.9 \mathrm{~cm})$ and larger mural nodule sizes (Height of mural nodules $>1.5 \mathrm{~cm}$ ), and were more likely to exhibit a taller than wider lesion (height-width ratio of mural nodules $>0.9$ ). 'Height of mural nodules $>1.5 \mathrm{~cm}$ ' was the most valuable predictor for discriminating EAOC from benign $\mathrm{OE}$ (AUC, 0.99; 95\% CI, 0.97-1.0; sensitivity 95.0\%, specificity 95.2\%).

The differential diagnosis between EAOC with predominantly cystic appearances and benign $\mathrm{OE}$ is difficult and require quantitative parameters that reflect the metabolic and biochemical state of cyst fluid, which are translated into disease diagnosis. When compared to subjects with benign $\mathrm{OE}$, cyst fluid analysis revealed low concentrations of $\mathrm{Hb}$ and iron-related compounds in EAOC (7). This observation reveals the possibility of early detection of biochemical changes before morphological variations are captured through ultrasonographic and MRI diagnosis. Investigators highlighted practical applications of measuring these metabolic and biochemical markers for discrimination between benign and malignant endometriosis (7-9). Since the cyst fluid sampling is not a serum-based test, noninvasive techniques for assessment of cyst fluid levels of $\mathrm{Hb}$-related compounds are clinically required. $\mathrm{Hb}$ displays absorption spectra in the visible and $\mathrm{UV}$ regions (10). The specific spectroscopic changes are invaluable for diagnostic applications of the concentration of $\mathrm{Hb}(10)$. Such novel diagnostic modalities include electronic absorption spectroscopy, near infrared approach and MR transverse relaxometry.

Identification of cyst fluid hemoglobin species by electronic absorption spectroscopy. Iwabuchi et al (11), reported the potential of electronic absorption spectroscopy for evaluating the biochemical changes through a metallobiologic

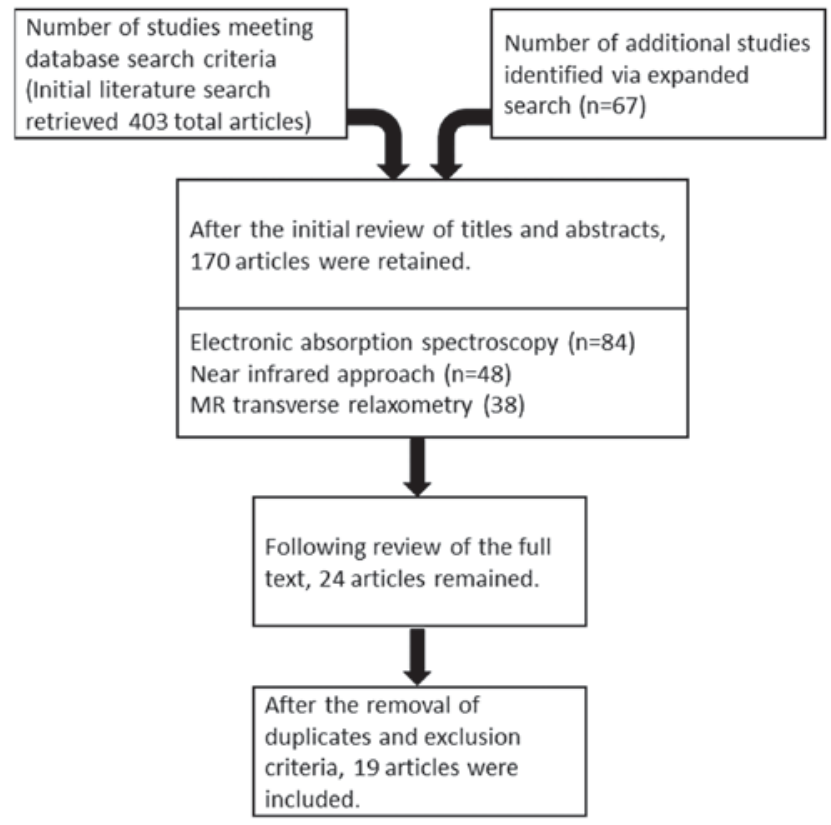

Figure 1. Flow chart of the literature search.

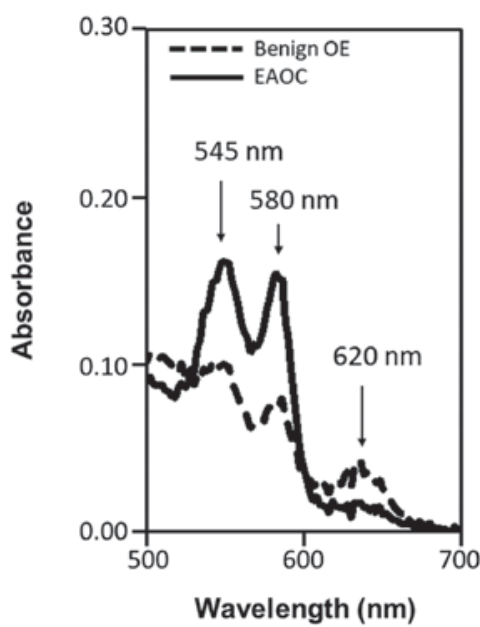

Figure 2. Absorption spectra of cyst fluid samples obtained from benign $\mathrm{OE}$ and EAOC. The ex-vivo cyst fluid samples were analyzed by absorption spectral mapping (11). The small-amplitude peak component of wavelength of $620 \mathrm{~nm}$ is specific for metHb. A difference was observed in the region of $620 \mathrm{~nm}$ between the absorption spectrum of benign cysts (dashed line) and that of EAOC (solid line). OE, ovarian endometrial; EAOC, endometriosis-associated ovarian cancer.

diagnosis. Absorbance based approaches have been developed for measuring $\mathrm{Hb}$ and iron-related compounds in biological samples (12). It is a well-known fact that a multi-wavelength spectrophotometric method provides a noninvasive and real-time measurement of $\mathrm{Hb}$ concentration (13). Fig. 2 shows representative absorption spectra of cyst fluid samples obtained from benign $\mathrm{OE}$ and EAOC. OxyHb is comprised of large-amplitude two peak components of wavelengths of 540 and $580 \mathrm{~nm}$, while metHb has strong absorption than oxyHb, from 610 to $630 \mathrm{~nm}$ (11). Absorption spectral curve of metHb shows one absorption peak at $620 \mathrm{~nm}$ (11). They hypothesized that the characteristic change at $620 \mathrm{~nm}$ or the $620 \mathrm{~nm} / 580 \mathrm{~nm}$ ratio would be used to determine relative 
A

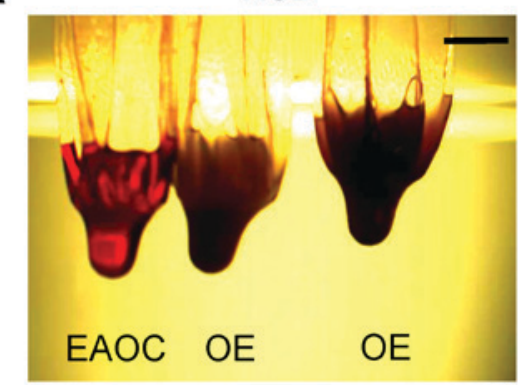

C

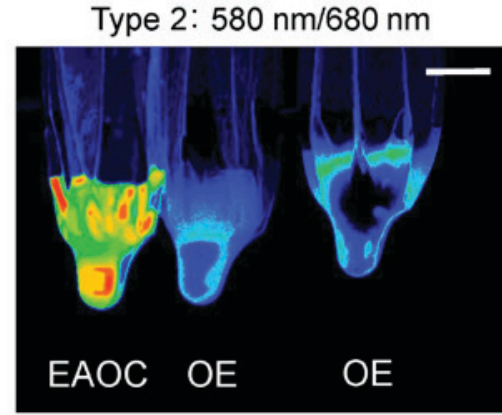

B

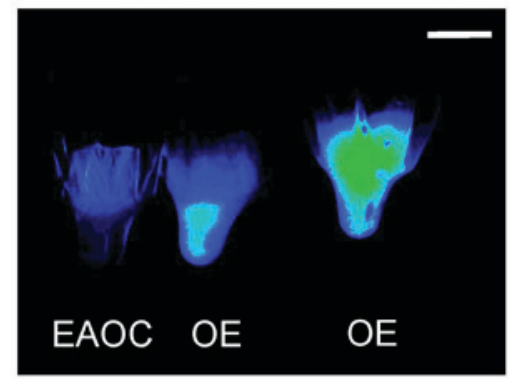

D

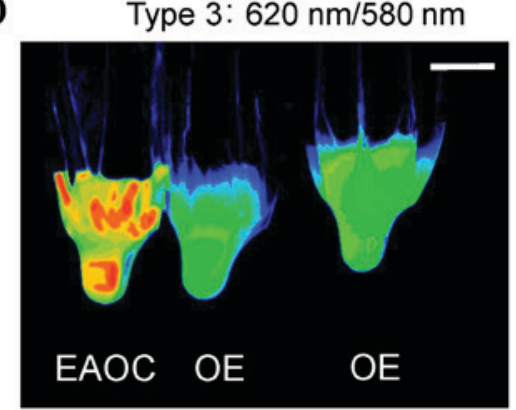

Figure 3. Optical images of cyst fluid samples obtained from benign OE and EAOC in an ex-vivo model. These pictures represent spectrophotometric evaluation of color images. Three patients (left, EAOC; middle, benign OE; and right, benign OE) were pathologically confirmed by routine diagnostic procedures. The cyst fluid sample was illuminated with light from halogen sources (A) passing through two types of band pass filter (wavelengths: (B) 620 and 680 nm; (C) 580 and $680 \mathrm{~nm}$; and (D) 620 and $580 \mathrm{~nm}$. Transmitted light was detected using a digital charge-coupled device (CCD) camera. A detailed description of sample loading into the condom, data analysis method, and other phenomena is described previously (11). Scale bar, $1 \mathrm{~cm}$. OE, ovarian endometrial; EAOC, endometriosis-associated ovarian cancer; RGB, red-green-blue.

metHb concentration or the metHb/oxyHb ratio, respectively. Relative concentrations of $\mathrm{Hb}$ species can be calculated from the absorption spectra. The 620/580 $\mathrm{nm}$ absorption ratio was significantly lower in the cyst fluid of patients with EAOC than in that of patients with benign cysts $(0.389 \pm 0.266$ vs. $0.666 \pm 0.188, P=0.021)(11)$. The sensitivity, specificity, positive predictive value, and negative predictive value for predicting EAOC were $62.5,100.0,100.0$, and $92.1 \%$, respectively (11). From this data, metHb is rich in the cyst fluid of $\mathrm{OE}$ and is a specific biomarker for discriminating EAOC from benign OE. Iwabuchi et al (11), reported that heme iron is abundant in the cyst fluid of benign $\mathrm{OE}$, therefore, autoxidation might be the main process accomplishing the MetHb increases: $\mathrm{Hb}-\mathrm{Fe}^{2+}$ (oxyHb) $+\mathrm{O}_{2} \rightarrow \mathrm{Hb}-\mathrm{Fe}^{3+}$ (metHb) $+\mathrm{O}_{2}^{-}$. Previous studies showed that the patients with EAOC had much lower levels of $\mathrm{Hb}$ and iron-related compounds compared with those with benign $\mathrm{OE}(7,8)$. EAOC allows the rapid reduction of metHb possibly through the conversion of metHb into oxyHb (in vivo autoreduction). Glutathione, an antioxidant overexpressed in EAOC, is shown to be responsible for the conversion of metHb to oxyHb $(14,15)$. The difference in the metHb-to-oxyHb ratio between benign and malignant endometriosis supports the hypothesis that this is not a consequence of the simple dilution of the cyst fluids.

Furthermore, these authors proposed that an optical method using light from halogen sources in a simple imaging setup would provide reliable visualization of metHb and oxyHb content in a cyst fluid sample. A simple and inexpensive optical imaging system is composed of three functional modules: The visible light stimulator, the image acquisition module with specific bandpass filters as a wavelength selector, and the image processing unit (11). Fig. 3 provides a representative ex-vivo color changes of the cyst fluid samples that could be distinguished by the human eye on any specimen (11). This optical imaging system could allow visual detection of color changes by monitoring specific wavelengths in the absorption spectra. Visible light exhibited the limited penetration depth in biological tissue, thus restricting the application of this optical imaging method for disease diagnosis.

Identification of cystfluidhemoglobin species bynear infrared approach. A near-infrared light can overcome the main limitation of visible light imaging, indicating beneficial effects in applications in humans. Kawahara et al (16), used noninvasive near-infrared spectrophotometric method to determine cyst fluid absorption and scattering coefficients and absolute cyst fluid $\mathrm{Hb}$ and total iron concentrations. This study provided the design, characterization, performance, and preclinical validation of a broadband near-infrared spectroscopic system for determining total iron concentration of a biological sample. The system is composed of five functional modules: the light stimulator, measuring instrument, photoelectric detection, the image acquisition module, and the image signal processing (Fig. 4A). They used cyst fluid samples obtained from surgery in an ex vivo setting. An aliquot of cyst fluid sample was transferred to a disposable cuvette. To estimate sufficient barrier penetration, the prepared cuvette was covered with a commercially available chicken with a different thickness $(0$, 5, 10 and $20 \mathrm{~mm}$ ) (Fig. 4B and C) (16). This model mimics the anatomical structure, including vaginal wall layer and tumor surface layer. The light reflected from each cyst fluid sample [change in luminance, $-\Delta \mathrm{l}\left(\mathrm{cd} / \mathrm{m}^{2}\right)$ ] was spectrally 


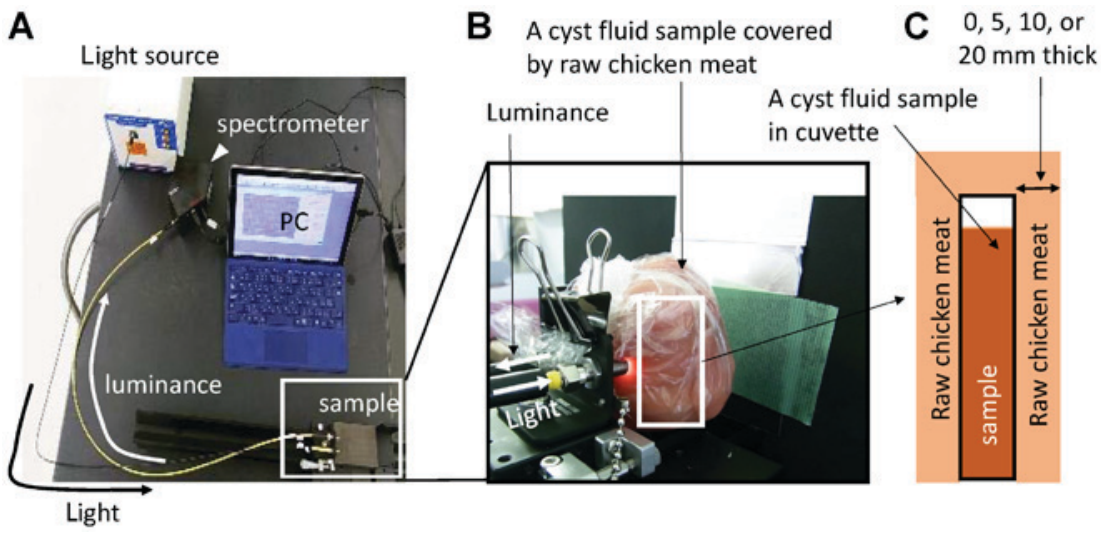

Figure 4. A near-infrared spectroscopic measurements using the ex-vivo cyst fluid samples. This picture provides photographic representations of the near-infrared spectroscopic imaging system. The near-infrared system has been developed to determine the total iron concentrations of each cyst fluid sample. (A) A complete view of the near-infrared spectroscopic imaging system. (B) The luminance were analyzed based on the reflection properties. (C) The prepared cuvette was covered by raw chicken meat $(2 \mathrm{~cm}$ thick). PC, personal computer.

measured by a CCD camera with a band-path filter $(800 \mathrm{~nm})$ via the optical spectroscopic imaging apparatus. The imaging results, $-\Delta \mathrm{l}$, for the ex-vivo specimens obtained with a near-infrared spectroscopy were compared to the results of total iron concentrations. The study uncovered that the change in luminance $\left(-\Delta \mathrm{l}, \mathrm{cd} / \mathrm{m}^{2}\right)$ of cystic fluid from the EAOC group was significantly higher compared with that of the OE group (Fig. 5). The- $\Delta$ l level exhibits significant negative correlation with total iron concentration and could serve as a simple, rapid and accurate method to discriminate EAOC from benign $\mathrm{OE}$, with high sensitivity $(85 \%)$ and specificity (95\%) (16). A $800 \mathrm{~nm}$ near infrared light reached the depth of $2 \mathrm{~cm}$ and delivered approximately $5 \%$ of the surface power density. This ex-vivo study provided a powerful near-infrared approach for quantitative discrimination of benign and malignant cyst fluids. Recent advances in the field of metallobiology would highlight the potential for future clinical application of near infrared approach.

At present, the cystic mass with mural nodules was virtually always surgically removed to exclude malignancy, which can lead to over-diagnosis and over-treatment. On the other hand, pre-malignant lesions without a mural nodule may pose a diagnostic challenge on routine ultrasonography. We believe that noninvasive near-infrared spectroscopy monitoring can help to identify women who should undergo surgery before the appearance of malignant morphological features in outpatient settings. More studies are needed for better characterization of its diagnostic value and potential for clinical application.

Identification of cyst fluid hemoglobin species by MR transverse relaxometry. There has been increasing interest in noninvasive assessment of tissue iron overload (17). For example, liver and heart iron content was reliably quantified by measurement of the transverse magnetic relaxation rate R2 or R2* value using complex chemical shift-encoded MR examination (18). Yoshimoto et al (9), hypothesized that the MR transverse relaxometry technique would be applied to detect the change of total iron concentration in endometriotic cyst fluid. This idea came from the prior studies showing a positive correlation between tissue iron levels and MR transverse relaxometry (17-20). They evaluated for the first

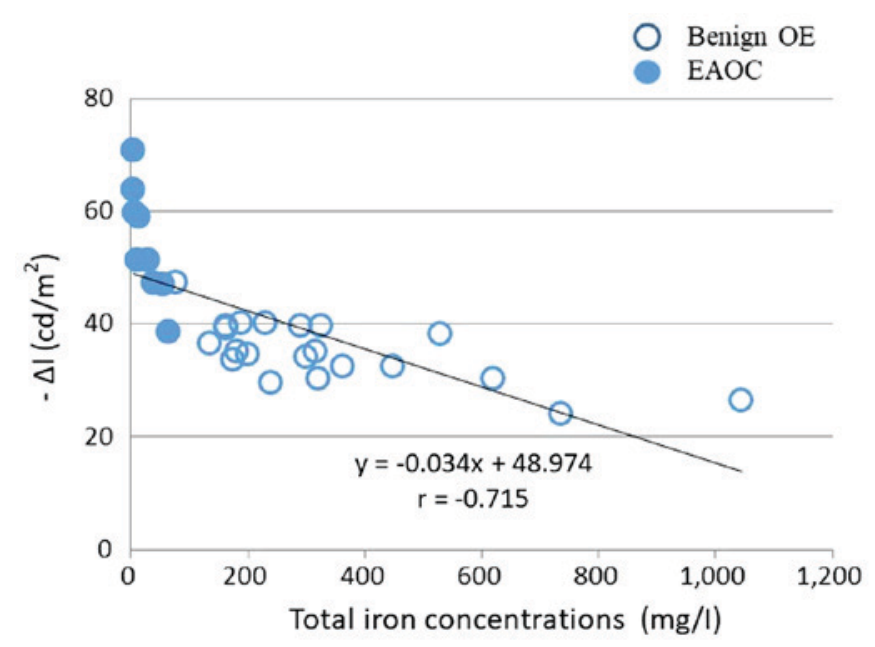

Figure 5. Correlation of $-\Delta \mathrm{l}\left(\mathrm{cd} / \mathrm{m}^{2}\right)$ and total iron concentration. Correlation analysis revealed negative correlation between $-\Delta \mathrm{l}\left(\mathrm{cd} / \mathrm{m}^{2}\right)$ and cyst fluid levels of total iron $(\mathrm{mg} / \mathrm{l})$. Change in luminance, $-\Delta \mathrm{l}\left[\mathrm{cd} / \mathrm{m}^{2}\right]$ was spectrally measured by a CCD camera with a band-path filter $(800 \mathrm{~nm})$ via the optical spectroscopic imaging apparatus. CCD, charge-coupled device; OE, ovarian endometrial; EAOC, endometriosis-associated ovarian cancer.

time the correlation between MR transverse relaxometry and the cyst fluid total iron, heme iron and free iron levels in patients with $\mathrm{OE}$ and its malignant tumor to assess whether this method can predict EAOC from benign OE (9). Fig. 6 represent conventional MR images (A-D) and MR transverse relaxometry images ( $\mathrm{E}$ and $\mathrm{F}$ ) created from the quantitative scan. The preliminary in vivo $\mathrm{R} 2$ value was 8 -fold lower in EAOC than in benign OE ( $\mathrm{G}$ and $\mathrm{H})$ (9). They draw attention to MR transverse relaxometry as important for the diagnosis of malignant transformation. In a prospective study at a single tertiary institution, Yoshimoto et al (9), determined and analyzed the R2 values by MR transverse relaxometry. Consequently, the in vivo $\mathrm{R} 2$ values in the EAOC group $(\mathrm{n}=12)$ were significantly lower than those in the benign $\mathrm{OE}$ group

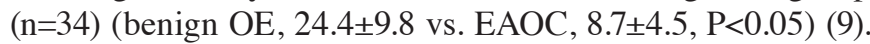
In the EAOC group, the in vivo $\mathrm{R} 2$ values decreased 2.7-fold compared to the benign OE group. The optimal predictive cutoff value was 12.1 , giving a sensitivity and specificity of 


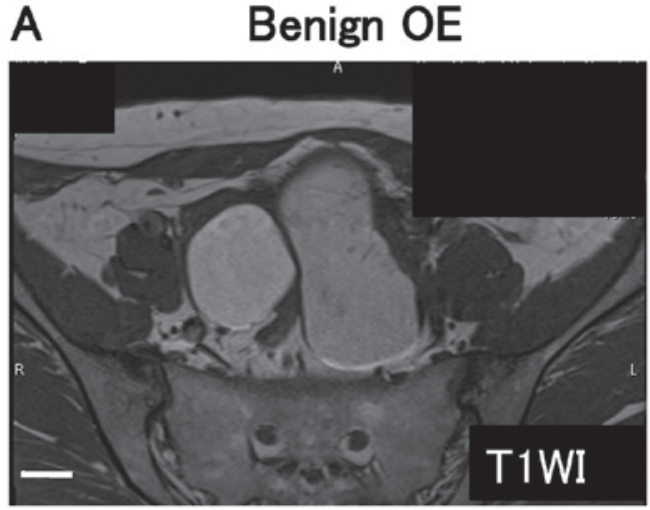

C

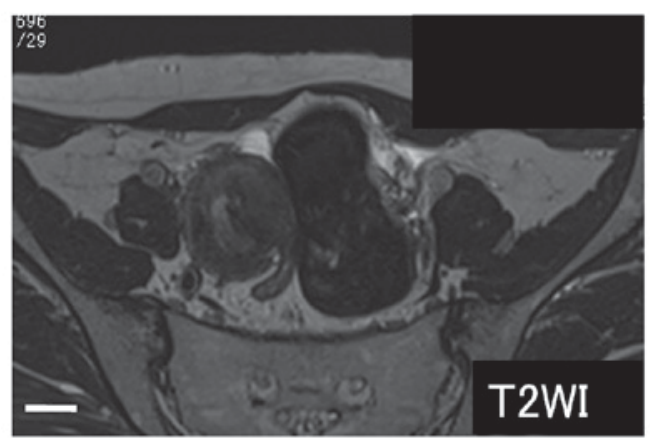

B

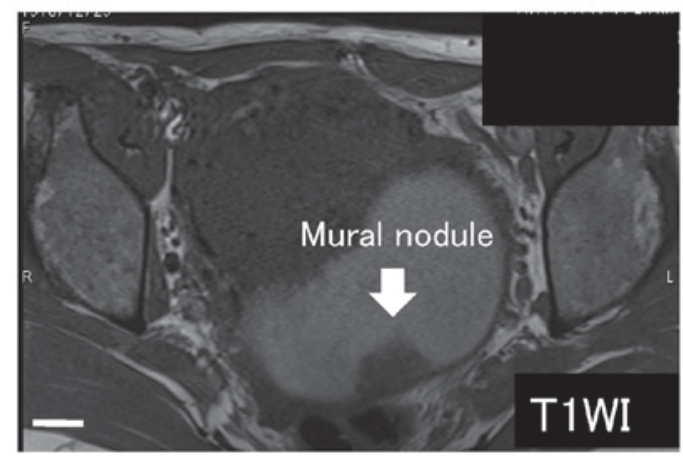

D

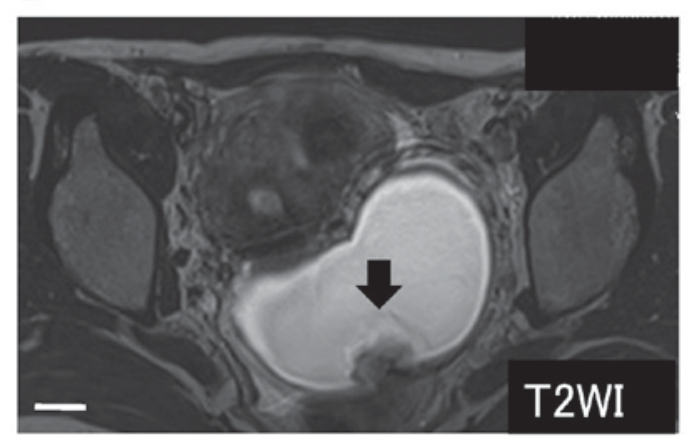

E

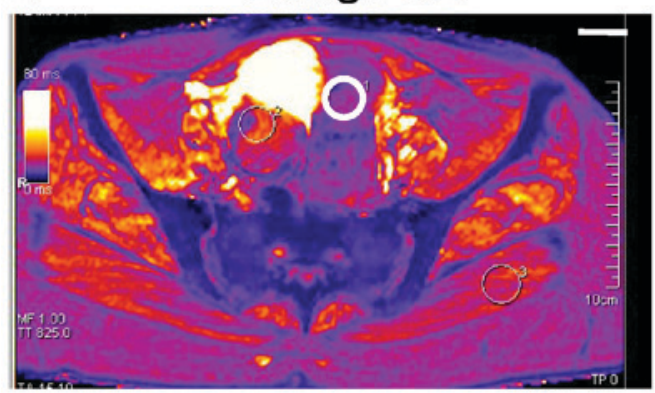

G

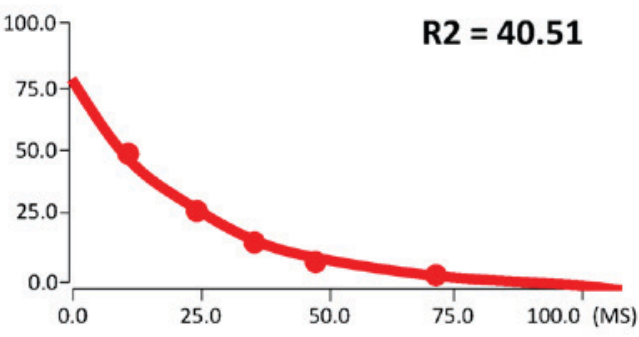

R2 maps

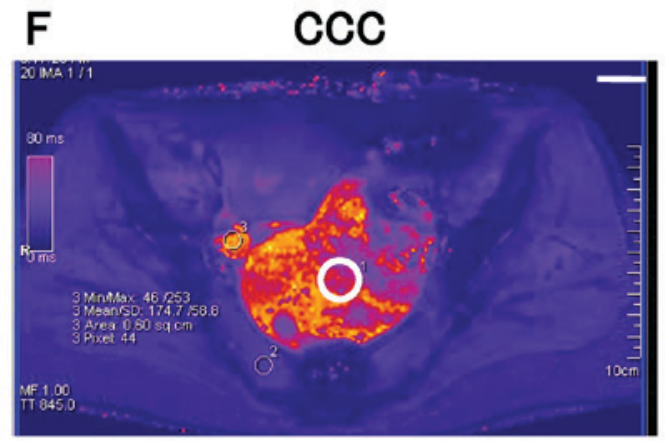

H

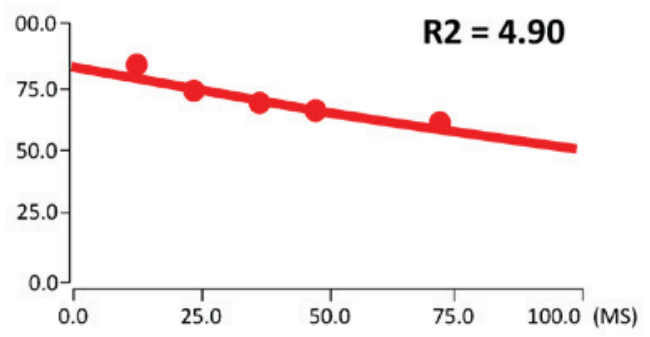

Figure 6. A direct comparison between conventional MR images and MR transverse relaxometry images. MR images represent an benign ovarian endometrioma in a 36-year-old woman (A, C, E and G) and a CCC in a 59-year-old woman (B, D, F and H). Axial T1WI (A) and T2WI (C) show a large, bilateral and unilocular well-defined endometriotic cystic mass. Axial T1WI (B) and T2WI (D) show a large, unilateral and unilocular well-defined malignant cystic mass with an irregular mural nodule. E and F are examples of MR transverse relaxometry in patients with benign OE and EAOC, respectively. A radiologist drew regions of interest (ROI, voxel) for the analysis. Images are subsequently processed to estimate the R2 relaxation rate at each voxel. In this technique, several spin-echo images are acquired with increasing echo times (ms). MR transverse relaxometry data is plotted regarding R2 as a function of the time. The bottom $\mathrm{G}$ and $\mathrm{H}$ show in vivo R2 relaxation time: The patient with benign $\mathrm{OE}(\mathrm{G}, \mathrm{R} 2=40.51)$ and the patient with EAOC $(\mathrm{H}, \mathrm{R} 2=4.90)$. Scale bar, $1 \mathrm{~cm}$. $\mathrm{CCC}$, clear cell carcinoma; T1WI, T1-weighted imaging; T2WI, T2-weighted imaging; ROI, region of interest; OE, ovarian endometrial.

86 and $94 \%$, respectively. Taken together, the presented MR relaxometry might be a noninvasive preoperative prediction tool and showed a favorable predictive accuracy for the malignant transformation, but the implementation of MR imaging in the outpatient clinic is occasionally difficult. Further clinical study and multicenter validation are needed to establish a non-invasive tool for early diagnosis and acquire high-level evidence for its clinical application. 


\section{Discussion}

The purpose of this review is to discuss the current and future available methods for diagnosis of malignant transformation of endometriosis. Noninvasive tests and candidate biomarkers of early detection of the malignant transformation are essential for disease monitoring and accurate diagnosis. Malignant cystic tumors, including EAOC, may be associated with worrisome features (e.g., mural nodule of various types and thick septa) on ultrasonography, CT and MR images. The presence of mural nodules is a potentially suitable marker for differentiating EAOC from benign OE, and is important for making decisions about surgical interventions. Cases of EAOC without mural nodules are very rare, because malignant transformation in its early stage usually remains undetected by conventional imaging modalities such as ultrasound, CT and MRI.

Currently available serum-based tests, including CA125, have been disappointing (4). Arakawa et al (21), reported that serum Tissue Factor Pathway Inhibitor-2 (TFPI2) levels were elevated only in patients with CCC among epithelial ovarian cancer. The preliminary study demonstrated that patients with endometriosis presented mild-to-moderate elevation of serum CA125 levels, but may be characterized by normal serum levels of TFPI2 (21). This study suggests that TFPI2 may be useful for discriminating CCC from benign OE. Further investigation of TFPI2 as the serum biomarker is encouraged.

Advances in understanding the pathogenesis of endometriosis malignancy, based on biological and biochemical concepts, have brought about new approaches in EAOC diagnosis and management. The levels of the $\mathrm{Hb}$ and iron-related compounds in the cyst fluid samples significantly changed between the benign $\mathrm{OE}$ and EAOC. We present a review of various noninvasive techniques being currently utilized in preclinical studies or being developed for future applications $(7,8)$. Imaging modalities discussed include electronic absorption spectroscopy, near infrared approach and MR transverse relaxometry. The diagnosis of EAOC might be more accurately achieved in combination of the conventional imaging methods and the use of novel noninvasive methods: i) Combination of transvaginal ultrasonography and near infrared approach and ii) combination of MRI and MR transverse relaxometry. A new device, consisting of transvaginal ultrasound-guided near infrared system (composite-type optical ultrasonography), will be developed to noninvasively measure the cyst fluid $\mathrm{Hb}$ levels that could be an important instrument for clinical use in an office setting. Long-term goal is to explore the clinical value of composite-type near infrared-based optical system in diagnosis of the malignant transformation of endometriosis. Considering conveniency for medical doctors, it is useful to use composite-type optical ultrasonography when they identify the morphological changes in benign OE. Such a device will make the early diagnosis more accurate. Noninvasive tests may allow for consideration of avoidance of diagnostic surgery and help to identify women who should undergo surgery before the appearance of malignant morphological features in outpatient settings.

The article will highlight recent efforts in multimodal imagings, including electronic absorption spectroscopy, near infrared metallobiology approach and MR transverse relaxometry, and discuss to provide an outlook on future research directions. To better understand the implications of pathogenesis, we require various techniques including clinical, morphological, and molecular assessments. In addition to standard anatomic MRI, MR relaxometry is a noninvasive imaging technique that can assess the iron concentration at the molecular level. In the near future, a comprehensive MRI study would provide quantification of not only iron, but also oxyhemoglobin and methemoglobin contents. In fact, the study by Iwabuchi et al (11), showed that electronic absorption spectroscopy can assess the relative concentrations of hemoglobin species. Therefore, there is increasing interest in the role of MR relaxometry for diagnosis of malignant transformation of endometriosis. To say the least, combining MR relaxometry with MRI may help in assessing the early diagnosis of malignant transformation. This may allow MR relaxometry to go beyond the current role of MRI.

In conclusion, special emphasis is given to recent advances in the imaging modalities for the noninvasive diagnosis of malignant transformation of endometriosis.

\section{Acknowledgements}

Not applicable.

\section{Funding}

The present study was supported by JSPS KAKENHI (grant no. JP16K11150) and Tohoku Bureau of Economy, Trade and Industry (Tohoku grant no. 1607028).

\section{Availability of data and materials}

All data generated or analyzed during the present study are included in this published article.

\section{Authors' contributions}

YY, NK and KO collected data regarding the epigenetic and genetic abnormalities, and underlying mechanism of endometriosis transformation using the PubMed database. NK, $\mathrm{KO}$ and $\mathrm{CY}$ performed the literature search and supervised the study. HK and CY made substantial contributions to the conception of the study. HK contributed to the study design and interpretation of included research studies. All authors read and approved the final version of the manuscript.

\section{Ethics approval and consent to participate}

Not applicable.

\section{Patient consent for publication}

Not applicable.

\section{Competing interests}

The authors declare that they have no competing interests. 


\section{References}

1. Kobayashi H, Sumimoto K, Kitanaka T, Yamada Y, Sado T, Sakata M, Yoshida S, Kawaguchi R, Kanayama S, Shigetomi H, et al: Ovarian endometrioma-risks factors of ovarian cancer development. Eur J Obstet Gynecol Reprod Biol 138: 187-193, 2008.

2. Brinton LA, Sakoda LC, Sherman ME, Frederiksen K, Kjaer SK, Graubard BI, Olsen JH and Mellemkjaer L: Relationship of benign gynecologic diseases to subsequent risk of ovarian and uterine tumors. Cancer Epidemiol Biomarkers Prev 14: 2929-2935, 2005.

3. Nicklin J, Janda M, Gebski V, Jobling T, Land R, Manolitsas T, McCartney A, Nascimento M, Perrin L, Baker JF, et al: The utility of serum CA-125 in predicting extra-uterine disease in apparent early-stage endometrial cancer. Int J Cancer 131: 885-890, 2012

4. Taniguchi F, Harada T, Kobayashi H, Hayashi K, Momoeda M and Terakawa N: Clinical characteristics of patients in Japan with ovarian cancer presumably arising from ovarian endometrioma. Gynecol Obstet Invest 77: 104-110, 2014

5. Tanase Y, Kawaguchi R, Takahama J and Kobayashi H: Factors that differentiate between endometriosis-associated ovarian cancer and benign ovarian endometriosis with mural nodules. Magn Reson Med Sci 17: 231-237, 2018.

6. Zhou Y and Hua KQ: Ovarian endometriosis: Risk factor analysis and prediction of malignant transformation. Prz Menopauzalny 17: 43-48, 2018.

7. Yoshimoto C, Iwabuchi T, Shigetomi $\mathrm{H}$ and Kobayashi $\mathrm{H}$ : Cyst fluid iron-related compounds as useful markers to distinguish malignant transformation from benign endometriotic cysts. Cancer Biomark 15: 493-439, 2015.

8. Yamaguchi K, Mandai M, Toyokuni S, Hamanishi J, Higuchi T, Takakura K and Fujii S: Contents of endometriotic cysts, especially the high concentration of free iron, are a possible cause of carcinogenesis in the cysts through the iron-induced persistent oxidative stress. Clin Cancer Res 14: 32-40, 2008.

9. Yoshimoto C, Takahama J, Iwabuchi T, Uchikoshi M, Shigetomi $\mathrm{H}$ and Kobayashi $\mathrm{H}$ : Transverse relaxation rate of cyst fluid can predict malignant transformation of ovarian endometriosis. Magn Reson Med Sci 16: 137-145, 2017.

10. Nagai M, Mizusawa N, Kitagawa T and Nagatomo S: A role of heme side-chains of human hemoglobin in its function revealed by circular dichroism and resonance Raman spectroscopy. Biophys Rev 10: 271-284, 2018.
11. Iwabuchi $\mathrm{T}$, Yoshimoto $\mathrm{C}$, Shigetomi $\mathrm{H}$ and Kobayashi $\mathrm{H}$ : Cyst fluid hemoglobin species in endometriosis and its malignant transformation: The role of metallobiology. Oncol Lett 11: 3384-3388, 2016.

12. Oh JY, Hamm J, Xu X, Genschmer K, Zhong M, Lebensburger J, Marques MB, Kerby JD, Pittet JF, Gaggar A and Patel RP: Absorbance and redox based approaches for measuring free heme and free hemoglobin in biological matrices. Redox Biol 9: 167-177, 2016.

13. Colquhoun DA, Forkin KT, Durieux ME and Thiele RH: Ability of the Masimo pulse CO-Oximeter to detect changes in hemoglobin. J Clin Monit Comput 26: 69-73, 2012.

14. Lopes-Coelho F, Gouveia-Fernandes S, Gonçalves LG, Nunes C, Faustino I, Silva F, Félix A, Pereira SA and Serpa J: HNF1 $\beta$ drives glutathione (GSH) synthesis underlying intrinsic carboplatin resistance of ovarian clear cell carcinoma (OCCC). Tumour Biol 37: 4813-4829, 2016.

15. Harris IS, Treloar AE, Inoue S, Sasaki M, Gorrini C, Lee KC, Yung KY, Brenner D, Knobbe-Thomsen CB, Cox MA, et al: Glutathione and thioredoxin antioxidant pathways synergize to drive cancer initiation and progression. Cancer Cell 27: 211-222, 2015.

16. Kawahara N, Yamada Y, Ito F, Hojo W, Iwabuchi T and Kobayashi H: Discrimination of malignant transformation from benign endometriosis using a near-infrared approach. Exp Ther Med 15: 3000-3005, 2018.

17. Fischer R and Harmatz PR: Non-invasive assessment of tissue iron overload. Hematology Am Soc Hematol Educ Program: 215-221, 2009.

18. Wood JC, Enriquez C, Ghugre N, Tyzka JM, Carson S, Nelson MD and Coates TD: MRI R2 and R2* mapping accurately estimates hepatic iron concentration in transfusion-dependent thalassemia and sickle cell disease patients. Blood 106: 1460-1465, 2005.

19. Argyropoulou MI and Astrakas L: MRI evaluation of tissue iron burden in patients with beta-thalassaemia major. Pediatr Radiol 37: 1191-1200, 2007.

20. Verlhac S, Morel M, Bernaudin F, Béchet S, Jung C and Vasile M: Liver iron overload assessment by MRI R2* relaxometry in highly transfused pediatric patients: An agreement and reproducibility study. Diagn Interv Imaging 96: 259-264, 2015.

21. Arakawa N, Kobayashi H, Yonemoto N, Masuishi Y, Ino Y, Shigetomi H, Furukawa N, Ohtake N, Miyagi Y, Hirahara F, et al: Clinical Significance of tissue factor pathway inhibitor 2, a serum biomarker candidate for ovarian clear cell carcinoma. PLoS One 11: e0165609, 2016. 